\title{
Betriebsnahe Tarifpolitik durch rückübertragbare Öffnungsklauseln
}

\author{
Holger Brecht-Heitzmann \\ Marcel Gröls
}

\begin{abstract}
Seit Jahren sehen sich die Gewerkschaften mit der Forderung nach einer Öffnung ihrer Flächentarifverträge zugunsten von betrieblichen Regelungen konfrontiert. Durch zahlreiche Öffnungen ihrer Tarifverträge haben sie hierauf reagiert, damit aber auch deren ordnende Wirkung immer stärker relativiert. Weitere Risiken einer Verlagerung der Regelungskompetenzen auf die Betriebe resultieren aus der bestehenden Machtasymmetrie zwischen Betriebsräten und Arbeitgebern. Es ist daher an der Zeit, mit einer neuen Form der Öffnungsklausel betriebsindividuelle Lösungen zu ermöglichen. Gelingt es den Betriebsparteien nicht, sich im Rahmen der tariflichen Vorgaben zu einigen, sollte diese Aufgabe zurück an die Tarifparteien fallen.
\end{abstract}

\section{Einleitung}

Mit dem Leber-Kompromiss in der Metallund Elektroindustrie von 1984 wurde ein Prozess eingeleitet, der mit dem Schlagwort „Verbetrieblichung" charakterisiert wird und den Bedeutungszuwachs der betrieblichen zulasten der tariflichen Ebene beschreibt. Dem Druck, den Flächentarifvertrag zugunsten betrieblicher Regelungen zu öffnen, wurde mit einer Vielzahl tariflicher Öffnungsklauseln entsprochen. Hierdurch weist das heutige Tarifsystem in Deutschland einen ausgesprochen hohen Grad an Flexibilität auf (Bispinck/WSITarifarchiv 2005).

Diese Öffnungen waren nicht zuletzt die Folge abweichender Regelungen auf betrieblicher Ebene, die eine präjudizierende Wirkung auf den Flächentarifvertrag ausüben: Mit Verweis auf Wettbewerbsverzerrungen werden vom Tarifvertrag abweichende Regelungen in einem Betrieb zum Argument für entsprechende Abweichungen in anderen Betrieben. Je mehr Betriebe jedoch tarifliche Vorgaben missachten, desto stärker büßt der Tarifvertrag seine ordnende Wirkung ein. So waren die Tarifvertragsparteien teilweise dazu gezwungen, dem Angleichungsdruck nachzugeben und abweichende Regelungen durch partielle Öffnungen ihrer Tarifverträge wieder zu legalisieren (Brecht 2003, S. 37ff.).

Jedoch ist auch eine solche begrenzte Öffnung mit nicht unerheblichen Risiken verbunden. Zum einen führt auch sie zu einer Verringerung der tariflichen Ordnungswirkung, die für gleiche Bedingungen in allen Betrieben der jeweiligen Branche sorgen soll. Zum anderen besteht eine Disparität zwischen den Betriebsparteien, da ein Arbeitgeber kraft seiner unternehmerischen Entscheidungsfreiheit Sachzwänge schaffen kann, mit denen selbst erfahrene und durchsetzungsstarke Betriebsräte erheblich unter Druck gesetzt werden können. Nicht zuletzt aufgrund des Verbots von Arbeitskämpfen auf betrieblicher Ebene besteht bei einer Öffnung des Tarifvertrags die Gefahr, dass für die Arbeitnehmer weniger günstige Vereinbarungen getroffen werden, als dies bei einer tariflichen Regelung der Fall wäre.

Die Tarifvertragsparteien haben versucht, durch eine kontrollierte Dezentralisierung diese Risiken zu minimieren. So sollen durch eine Verzahnung von betrieblicher und tariflicher Rechtsetzung Einflussmöglichkeiten der Tarifparteien gewahrt bleiben. Korridorlösungen eröffnen einen klar definierten Rahmen, innerhalb dessen betriebliche Regelungen möglich sind. Diese Ansätze können weiter entwickelt werden, um das Zusammenspiel betrieblicher und tariflicher Rechtsetzung zu optimieren. Im Folgenden soll dazu ein neues tarifliches Modell vorgestellt werden, das als rückübertragbare Öffnungsklausel bezeichnet wird. Zu untersuchen ist hierbei zum einen die rechtliche Zulässigkeit einer derartigen tariflichen Regelung. Zum anderen soll die Attraktivität eines derartigen Modells aus dem Blickwinkel der Beteiligten beleuchtet werden.

\section{Rückübertragbare Öffnungsklausel}

Eine wirksame Lösung für die beschriebenen Probleme könnte darin liegen, die er- probten Instrumente Öffnungsklausel und Ergänzungstarifvertrag intelligent zu kombinieren. Das Ziel ist, deren jeweilige Vorteile wirksam zu bündeln und die Nachteile weitgehend zu eliminieren. Es muss dazu, wie im Folgenden herausgearbeitet wird, eine Öffnungsklausel formuliert werden, in welcher sich die Tarifparteien die Möglichkeit vorbehalten, unter bestimmten Umständen eine Rückverlagerung der Verhandlungskompetenz in die Wege zu leiten. Es geht sozusagen um eine Öffnungsklausel mit Rückfahrschein. Wie bei der klassischen, unbeschränkten Öffnungsklausel würden die Tarifpartner den Betriebsparteien echte Verhandlungskompetenzen ermöglichen. Zweckmäßig wäre in diesem Fall ein Rahmen, sei es in Form eines Korridors oder auf andere Weise. Dies würde den betrieblichen Akteuren die Gelegenheit geben, unter Aufbietung aller erprobten formellen und informellen innerbetrieblichen Verhandlungswege zu einer einvernehmlichen Lösung zu gelangen. Den Betriebsparteien würde das Recht eingeräumt, in einem vordefinierten, überschaubaren Zeitraum zu einer sachgerechten ergänzenden Betriebsvereinbarung zu gelangen. Ihnen würde dabei nicht die volle

Holger Brecht-Heitzmann, Prof. Dr., Junior-
professor an der Universität Hamburg.
Arbeitsschwerpunkte: Arbeitsrecht,
Zivilrecht, Unternehmensmitbestimmung.
e-mail: Holger.Brecht-Heitzmann@wiso.uni-
hamburg.de
Marcel Gröls, B. A. in Wirtschafts- und
Arbeitsrecht, Universität Hamburg.
Arbeitsschwerpunkte: Arbeitsrecht, Personal-
wirtschaft.
e-mail: marcel.groels@goog/email.com

Holger Brecht-Heitzmann, Prof. Dr., Juniorprofessor an der Universität Hamburg. Arbeitsschwerpunkte: Arbeitsrecht Zivilrecht, Unternehmensmitbestimmung. e-mail: Holger.Brecht-Heitzmann@wiso.unihamburg.de Arbeitsrecht, Universität Hamburg. wirtschaft.

e-mail: marcel.groels@goog/email.com 
Last des Einigungsdrucks aufgebürdet, denn wenn eine Einigung nicht gelingt, fällt die diesbezügliche Normsetzungskompetenz an die Tarifvertragsparteien zurück, die in diesem Fall keiner Friedenspflicht mehr unterlägen. Die Tarifpartner streben in diesem Fall ihrerseits einen entsprechenden Haustarifvertrag oder auch einen betriebsbezogenen Verbandstarifvertrag an . In beiden Szenarien geht es gezielt um eine betriebsindividuelle Lösung. Der Unterschied zu Öffnungsklauseln, bei denen sich die Tarifpartner nur die letztgültige $\mathrm{Zu}$ stimmung vorbehalten, liegt insbesondere darin, dass noch einmal Nachverhandlungen der Tarifvertragsparteien mit allen Kampfmitteln möglich sind. An dieser Stelle sei daran erinnert, dass Haustarifverträge Tarifverträge sind, welche die zuständige Gewerkschaft direkt mit dem jeweiligen Arbeitgeber abschließt. Der Begriff Firmentarifvertrag wird synonym verwendet. Hingegen besteht die Eigenart firmenbezogener Verbandstarifverträge oder Ergänzungstarifverträge darin, dass in diesem Fall die Gewerkschaft mit dem Arbeitgeberverband abschließt - allerdings nicht auf die Branche bezogen, sondern auf ein einzelnes Verbandsmitglied (Ohl et al. 1997, S. 42ff.). Für das vorliegend vorgestellte Modell sind zunächst prinzipiell beide Varianten denkbar, wenngleich es bei näherer Betrachtung einige Vorteile zugunsten des firmenbezogenen Verbandstarifvertrages geben mag. Der besseren Lesbarkeit halber wird das hier vorgeschlagene Vorgehen im Folgenden verkürzt als rückübertragbare Öffnungsklausel bezeichnet.

Die Tarifpartner würden also den betrieblichen Akteuren zunächst einmal eine echte Chance einräumen, unter Aufbietung ihrer - oft bewährten - Gesprächskultur zu einer maßgeschneiderten Lösung zu gelangen. Dennoch würden sie weder die Entscheidungskompetenz endgültig aus der Hand legen noch die Betriebspartner im Konfliktfall weitgehend allein lassen, wie das klassische Öffnungsklauseln oftmals mit sich bringen. Denn den Betriebsparteien steht als Ultima ratio lediglich das mitunter zweischneidige Schwert einer Anrufung der Einigungsstelle zur Verfügung. Angesichts der Schlüsselstellung des Einigungsstellenvorsitzenden fällt dann eine wesentliche Entscheidungskompetenz an eine externe Person. Nach herrschender Meinung besteht - insbesondere in kleineren und mittleren Betrieben - das Risiko eines strukturellen Ungleichgewichts zwi- schen Betriebsrat und Arbeitgeber (Brecht 2003, S. 112ff.). Diese Disparität wird zwar durch die Einigungsstelle spürbar abgemildert, jedoch keineswegs ausgeglichen.

\section{Rechtliche Zulässigkeit}

Vor einer Betrachtung der Praktikabilität rückübertragbarer Öffnungsklauseln sollen zunächst die rechtsrelevanten Fragen mit einer solchen Regelung untersucht werden.

\subsection{NEGATIVE KOALITIONSFREIHEIT}

Teilweise wird angenommen, dass die Grundlage von Betriebsvereinbarungen, die auf tariflichen Öffnungsklauseln basieren, die Delegation von Rechtsetzungsbefugnissen ist: von den Tarifvertragsparteien hin $\mathrm{zu}$ den Betriebsparteien (vgl. Baumann 1992, S. 90ff.). Darauf aufbauend ist es nur noch ein kleiner Schritt anzunehmen, dass auf diesem Wege die negative Koalitionsfreiheit jener Betriebsangehörigen verletzt werden kann, die gar nicht Mitglied der tarifgebenden Gewerkschaft sind und nun aber dennoch über die Betriebsvereinbarung mittelbar von den tariflichen Vorgaben erfasst werden. Die Tarifpartner würden aus diesem Blickwinkel Rechte übertragen, über die sie selbst nicht verfügen.

Diese Sichtweise wird der Regelungssystematik der dualen Struktur der Interessenvertretung in Deutschland allerdings nicht gerecht: Wenn die Regelungssperre des $\S 77$ Abs. 3 BetrVG beseitigt wird, werden die Betriebsparteien wieder in den Stand gesetzt, als ob kein Tarifvertrag sie am Abschluss einer Betriebsvereinbarung hindern würde (Gragert 1997, S. 119). Die Betriebsparteien handeln dann im Rahmen ihrer originären Rechtsetzungsbefugnis und vertreten natürlich alle Beschäftigten einschließlich der nicht der tarifschließenden Gewerkschaft angehörenden „Außenseiter“, denn sie werden ja von der Gesamtheit der Beschäftigten gewählt. Der Arbeitnehmer wird in diesem Fall, wie das Bundesarbeitsgericht zutreffend feststellte, nicht vom Tarifvertrag, sondern von der normativen Wirkung der Betriebsvereinbarung erfasst (BAG 18.8.1987,AP Nr. 23 zu § 77 BetrVG).

Eine Verletzung der negativen Koalitionsfreiheit im Rahmen der vorgeschlagenen rückübertragbaren Öffnungsklausel lässt sich damit aus zwei Gründen vernei- nen: Zum einen sind die Betriebsparteien aufgrund der Regelungskompetenz, die die Öffnungsklausel definiert und gewährt, frei in ihrer Entscheidung, ob sie diesen Handlungsspielraum tatsächlich nutzen wollen. Eine solche Betriebsvereinbarung kann nicht gegen den Willen einer Betriebspartei durchgesetzt werden. Zum anderen bewirkt das vorgeschlagene Konfliktlösungsinstrument einer Rückübertragung an die Tarifvertragsparteien ohnehin nur eine Erfassung der tarifgebundenen Beschäftigten, sodass hierdurch die negative Koalitionsfreiheit der Außenseiter gar nicht beeinträchtigt werden kann.

\subsection{ORDNUNGSAUFGABE DER TARIFVERTRAGSPARTEIEN?}

Vereinzelt wird behauptet, Öffnungsklauseln wie auch Öffnungen für Ergänzungstarifverträge seien nicht mit der Ordnungsfunktion des Tarifvertrages zu vereinbaren (Matthes 1998, S. 483). Der Begriff der Ordnungsfunktion wird in diesem Zusammenhang nicht einheitlich definiert. Wenn das Bundesverfassungsgericht den Zweck der Koalitionsfreiheit darin sieht, „das Arbeitsleben im einzelnen durch Tarifverträge sinnvoll zu ordnen, insbesondere die Höhe der Arbeitsvergütung für die verschiedenen Berufstätigkeiten festzulegen und so letztlich die Gemeinschaft sozial zu befrieden" (BVerfG 6.5.1964, NJW 1964, S. 1267,1268$)$, so folgt daraus für einige $\mathrm{Au}-$ toren eine umfassende und verpflichtende Ordnungsaufgabe der Koalitionspartner (Wiedemann 2007, Einleitung Rn. 17 mit weiteren Nennungen). Eine solche Annahme führt jedoch zu einer Tarifzensur, wenn einzelne Tarifverträge einer gerichtlichen Überprüfung in Hinblick auf ihre Vereinbarkeit mit einer so definierten Ordnungsfunktion unterzogen würden. Schon deshalb kann diese Auffassung keinen Bestand haben. Die Tarifautonomie gestattet es den Tarifparteien, im Rahmen eines kollektiven und autonomen Aushandlungsprozesses zur sachlich besten Lösung zu gelangen. Dem Staat und auch den Gerichten ist es damit notwendigerweise verboten, ihre eigenen Richtigkeitsvorstellungen anstelle der Tarifvertragsparteien zu setzen. Die Ordnungsfunktion umrahmt also nicht die inneren Grenzen der Tarifautonomie, sondern umschreibt vielmehr die innere Legitimation des Tarifsystems sowie dessen Auswirkungen auf den Arbeitsmarkt (Wendeling-Schröder 1998, S. 627). 


\subsection{UMFASSENDE REGELUNGSPFLICHT?}

Ähnliche Bedenken könnten bei Annahme einer umfassenden Regelungspflicht der Tarifparteien entstehen. Nach dieser Ansicht wäre es den Koalitionären selbst zwar gestattet, betriebliche Differenzierungen zu vereinbaren. Sie könnten sich jedoch kaum zugunsten der Betriebsparteien zurücknehmen, etwa indem sie durch Öffnungsklauseln Raum für eine Betriebsvereinbarung lassen. Kämen die Adressaten einer solchen Regelungspflicht nicht nach, könnte die Durchsetzungsmacht nach dieser Lesart verlorengehen, sodass die Tarifautonomie von innen heraus beschädigt würde.

Das Bundesarbeitsgericht (BAG) hat die Frage im Zusammenhang mit der Leber-Entscheidung angesprochen und diesbezüglich von einem Selbstentäußerungsverbot gesprochen, andere sprechen vom „Abdanken“ der Tarifvertragspartner. Insbesondere entschied das BAG, dass die materiellen Arbeitsbedingungen von den Tarifpartnern so umgrenzt werden müssen, dass den betrieblichen Beteiligten ein verhältnismäßig geringer Spielraum verbleibe (BAG 18.8.1987, AP Nr. 23 zu $\$ 77$ BetrVG 1972). Es obliege den Tarifparteien demnach, einen Grundbestand an Normen festzulegen (Schwarze 1990, S. 91f.). In der Literatur wird zudem $₫ 77$ Abs. 3 BetrVG als Begründung hinzugezogen: Wegen der dort genannten „ergänzenden“ Betriebsvereinbarung sei der gesetzgeberische Wille anzuerkennen, dass zumindest irgendeine Regelung getroffen werde. Dieser Norm komme insoweit einige Bedeutung $\mathrm{zu}$, da sie als Ausdruck der verfassungsrechtlichen Gewährleistung der Tarifautonomie zu interpretieren sei (Gragert 1997, S. 113).

Aus Art. 9 Abs. 3 GG ergibt sich jedoch keineswegs eine allumfassende Regelungspflicht, und zwar weder aus dem Wortlaut, noch aus der Systematik - sind doch Grundrechte klassischerweise Abwehrrechte gegen staatliche Eingriffe und begründen typischerweise keine Pflichten der Grundrechtsträger. Gerade die selbst gewählte Entscheidung der Tarifpartner, Sperrwirkungen zu beseitigen, ist folgerichtig als Ausdruck tarifautonomer Regelungsbefugnis anzusehen. Die rückübertragbare Öffnungsklausel öffnet nur einen genau umfassten Teilbereich zugunsten der betrieblichen Akteure und sieht zudem gegebenenfalls sogar eine Rückübertragung vor. Wenn für einen bestimmten Rahmen eine endgültige Öffnung der betrieblichen Regelungskompetenz möglich wäre, so muss eine Rückübertragung erst recht zulässig sein. Das vom BAG angenommene Selbstentäußerungsverbot kann folglich durch rückübertragbare Öffnungsklauseln nicht verletzt werden.

\subsection{FRIEDENSPFLICHT}

Von Bedeutung ist zudem die Frage, ob die rückübertragbare Öffnungsklausel zugleich Arbeitskampfmaßnahmen der Tarifpartner wieder ermöglichen darf. Insbesondere die Reichweite der Friedenspflicht verdient in diesem Zusammenhang eine nähere Betrachtung. Die Rechtsprechung geht davon aus, dass dem Tarifvertrag auch ohne explizite Vereinbarung eine relative Friedenspflicht innewohnt; denn nur so kann die Funktion der Tarifautonomie, die Arbeits- und Wirtschaftsbedingungen zu regeln, erfüllt werden. Die Friedenspflicht gewährleistet also Rechtssicherheit und Kalkulierbarkeit. Sie ist insofern relativ, als sich die Pflicht zum Verzicht auf Arbeitskampfmaßnahmen gleichwohl nur auf die vereinbarten Materien des Tarifvertrages bezieht: Für diese Bereiche muss sich das einzelne Unternehmen darauf verlassen können, nicht noch einmal zusätzlich zu „Nachverhandlungen“ herangezogen zu werden.

Es entspricht sowohl der herrschenden Meinung als auch der ständigen Rechtsprechung, dass es den Tarifvertragsparteien als Autoren der Tarifverträge zusteht, die relative Friedenspflicht inhaltlich, zeitlich und gegenständlich $\mathrm{zu}$ bestimmen (Otto in: Richardi/Wlotzke 2000, $\$ 285$ Rn. 81f.). Ihnen allein obliegt es, die Reichweite der Friedenspflicht festzulegen. Im Rahmen einer rückübertragbaren Öffnungsklausel würde der Tarifvertrag im offengelassenen Korridor explizit als nicht abgeschlossen betrachtet. Mit der Friedenspflicht kann daher nur dann ernstlich argumentiert werden, wenn Tarifverträge einen bestimmten Gegenstandsbereich abschließend geregelt haben und gleichwohl eine betriebliche Nachverhandlung angestrebt wird (Stein in: Kempen/Zachert 2006, \$2 Rn. 133). Ein solches Streben müsste dann tatsächlich als Verstoß gegen die Friedenspflicht betrachtet werden.

Für die rückübertragbare Öffnungsklausel gilt nun in der Tat eine partielle Friedenspflicht: Zum einen für den bereits ausgehandelten Grundbestand des Flächen- tarifvertrags. Auch was den betriebsindividuellen Einigungsrahmen angeht, so ergibt sich für den Zeitraum, der den betrieblichen Akteuren zur Einigung zur Verfügung gestellt wird, aus der Systematik der rückübertragbaren Öffnungsklausel zwingend eine Friedenspflicht. Diese endet jedoch, wenn keine Einigung zustande kommt und eine Rückdelegation an die Tarifparteien erfolgt. Das ergibt sich nicht nur obligat aus dem Wortlaut der Vereinbarung, in der ein entsprechender Passus aufgenommen werden sollte, sondern auch aus der tariflichen Systematik. Denn mit der Rückübertragung werden die Tarifparteien in den Stand versetzt, als ob nie ein betriebliches Zwischenspiel erfolgt wäre. Somit leben die Rechte und Pflichten der ursprünglichen Partner wieder vollumfänglich auf.

\subsection{VERBANDSRECHTLICHE BESCHRÄNKUNGEN?}

Gerade im Zusammenhang mit der Friedenspflicht stellt sich schließlich die Frage, ob ein Unternehmen das dem Arbeitgeberverband angehört, mit dem Ziel bestreikt werden darf, einen Haustarifvertrag oder unternehmensbezogenen Verbandstarifvertrag zu erzwingen. Das Bestehen einer lediglich relativen Friedenspflicht führt konsequenterweise dazu, dass ein derartiger Streik als zulässig angesehen werden muss.

Eine Mindermeinung führt gegen die Möglichkeit eines Streiks bei einem verbandsgebundenen Unternehmen in erster Linie den Schutzzweck der Verbandsbindung ins Felde: Der Arbeitgeber müsse durch seine Mitgliedschaft im Arbeitgeberverband grundsätzlich dagegen gesichert sein, über den Flächentarifvertrag hinaus noch individuell von der Gewerkschaft in Anspruch genommen zu werden (Bauer/ Krieger 2004, S. 1019ff.). Dem muss entgegengehalten werden, dass aus $\$ 2$ Abs. 1 TVG unmissverständlich die Tariffähigkeit des einzelnen Arbeitgebers folgt. Das BAG hat in einer Grundsatzentscheidung im Jahre 2002 festgestellt, dass neben dem Wortlaut auch Sinn und Zweck des $\$ 2$ Abs. 1 TVG nicht dessen teleologische Restriktion gebieten. „Zutreffend“, so das BAG, ,ist zwar, dass die dem einzelnen Arbeitgeber in $\$ 2$ Abs. 1 TVG zuerkannte Tariffähigkeit der effektiven Verwirklichung der Tarifautonomie dient, indem sie verhindert, dass sich der Arbeitgeber durch Fernbleiben von oder Austritt aus einem 
Verband tarifunfähig macht und sich so der Inanspruchnahme auf den Abschluss von Tarifverträgen entzieht. Dieser Zweck entfällt daher, wenn der Arbeitgeber Mitglied eines tarifwilligen Arbeitgeberverbands ist. Gleichwohl rechtfertigt dies nicht die Reduktion des $\$ 2$ Abs. 1 TVG. Denn dessen Zweck erschöpft sich nicht darin, für die Gewerkschaft einen Verhandlungsgegner bereitzustellen." Wie das Gericht weiter ausführt, ergibt sich die Tariffähigkeit des einzelnen Arbeitgebers aus dessen Betätigungsfreiheit nach Art. 9 Abs. 3 GG. Würde er durch seine Verbandszugehörigkeit die Tariffähigkeit verlieren, wäre diese derart eingeschränkt, dass nicht einmal freiwillige Vereinbarungen mit der Gewerkschaft möglich wären (BAG 10.12.2002, AP Nr. 162 zu Art. 9 GG Arbeitskampf).

Andere argumentieren, die Koalitionsfreiheit des Arbeitgeberverbandes sei gefährdet, wenn sich die einzelnen Unternehmen trotz Verbandszugehörigkeit immer noch selbst mit der Gewerkschaft auseinanderzusetzen hätten (so bereits Buchner 1970, S. 2074ff.). Dieser Gedanke greift für die vorliegende Konstellation schon deshalb nicht, weil der einzelne Arbeitgeber angesichts der explizit vereinbarten Öffnungsklausel durchaus keine Regelung hat, auf deren Geltung er vertrauen müsste. Es kommt nur noch in einem engen Rahmen zu Verhandlungen. Die meisten Bestandteile werden regelmäßig durch den flächendeckend vereinbarten Tarifvertrag geregelt sein. Im Übrigen wäre es ein Wertungswiderspruch, wenn die Gegner der Ergänzungs- oder Haustarifverträge Tariföffnungen für Betriebsvereinbarungen anerkennen, die unter dem Schutz des Art. 9 Abs. 3 GG stehenden Tarifverträge jedoch nicht (Wendeling-Schröder 1998, S. 627).

Auch der Gleichheitsgedanke stellt in diesem Zusammenhang keine Barriere für eine betriebsnahe Tarifpolitik dar. Diesbezüglich argumentieren manche, Arbeitgeberverbände hätten kein Recht unterschiedliche Regelungen zu vereinbaren, vielmehr müssten sie für eine Gleichbehandlung ihrer Mitglieder sorgen (Krichel 1986, S. 731ff.). Eine solche Ansicht verkennt jedoch zum einen, dass Beschränkungen aus dem verbandsrechtlichen Innenverhältnis sich nicht auf die Wirksamkeit des Handelns des Arbeitsgeberverbands im Außenverhältnis durch Abschluss eines firmenbezogenen Verbandstarifvertrags auswirken können (Brecht-Heitzmann 2007, S. 3618). Zum anderen unter- sagt Art. 3 GG lediglich sachlich nicht gerechtfertigte Differenzierungen. Betriebsbezogene und maßgeschneiderte Abschlüsse stellen aber keine willkürlichen, sondern im Gegenteil höchst planvolle und durch die konkrete Situation des betroffenen Betriebes sachlich gerechtfertigte Unterscheidungen dar. Darüber hinaus durchlaufen auch Haus- oder Ergänzungstarifverträge einen Aushandlungsprozess. Diesen unter verbandsrechtlichen Gesichtspunkten prüfen zu wollen, liefe für die Gerichte notwendig auf eine unzulässige Tarifzensur hinaus.

Schließlich werden noch satzungsrechtliche Aspekte gegen unternehmensbezogene Arbeitskämpfe angeführt. Wenn nach der Satzung des Arbeitgeberverbandes der Abschluss von Firmentarifverträgen durch ein Mitgliedsunternehmen ausgeschlossen sei, so stünde dieses Unternehmen als Verhandlungspartner nicht mehr zur Verfügung (vgl. Lobinger 2006, S. 20f.). Diesen Gedanken weist das BAG zu Recht zurück. Nach Meinung des Gerichts begründet die Satzung lediglich verbandsinterne Pflichten. Sie vermag jedoch nicht das Außenverhältnis der Mitglieder zu Dritten in der Weise zu gestalten, dass Arbeitgeber einem Streik einfach dadurch die Legitimität nehmen, dass sie sich Dritten gegenüber dazu verpflichten, keine Tarifverträge abzuschließen. Nach Ansicht des BAG kann ,der einzelne Arbeitgeber seine Tariffähigkeit nicht durch schuldrechtliche Verpflichtungen beseitigen, die er gegenüber einem Arbeitgeberverband eingeht. Er ist rechtlich nicht in der Lage, über seine Tariffähigkeit zu disponieren. Diese ist nicht nur ein Recht, sondern zugleich eine dem Arbeitgeber auch im Interesse des sozialen Gegenspielers gesetzlich verliehene, unverzichtbare Eigenschaft" (BAG, 10.12.2002, AP Nr. 162 zu Art. 9 GG Arbeitskampf).

\subsection{ZWISCHENERGEBNIS}

Zusammenfassend lässt sich festhalten, dass das eingangs vorgestellte Modell der rückübertragbaren Öffnungsklausel unter rechtlichen Gesichtspunkten einwandfrei implementierbar ist. Weder die richtig verstandene Ordnungsfunktion der Tarifparteien noch deren Verbot einer allzu weitgehenden Selbstentäußerung stellen ein Hindernis dar. Auch die Berücksichtigung der negativen Koalitionsfreiheit der Außenseiter schränkt eine solche betriebsnahe Tarifpolitik nicht ein. Sowohl die herrschende
Meinung als auch die Rechtsprechung verneinen eine Verletzung der negativen Koalitionsfreiheit selbst bei einer endgültigen Öffnung des Tarifvertrags. Um so mehr muss dies dann für ein Modell gelten, welches im Konfliktfall eine Rückdelegation vorsieht und insoweit sogar weniger weitgehend ist. Ferner ergeben sich aus Wortlaut und Systematik der rückübertragbaren Öffnungsklausel keine Konflikte mit der Friedenspflicht. Diese ist relativ, denn sie umfasst lediglich die bereits abschlieBend verhandelten Bestandteile des Tarifvertrages. Schließlich resultiert aus der Betätigungsfreiheit des Art. 9 Abs. 3 GG sowie der Regelung des $\$ 2$ Abs. 1 TVG die Fähigkeit des einzelnen Arbeitgebers, im Rahmen von Haustarifverträgen direkte Vereinbarungen mit der Gewerkschaft zu treffen. Auf eventuell einschränkende Aussagen, welche die Verbandssatzung seines Arbeitgeberverbandes enthalten mag, kommt es dabei nicht an. Der einzelne Arbeitgeber kann seine Tariffähigkeit nicht einfach durch schuldrechtliche Verpflichtungen beseitigen, die er gegenüber seinem Verband eingeht.

\section{Praktikabilität}

Nach der bejahten rechtlichen Machbarkeit rückübertragbarer Öffnungsklauseln stellt sich nun die Frage ihrer Praktikabilität und Attraktivität. Hierzu sollen nachfolgend die Interessen der jeweiligen Akteure analysiert werden.

\subsection{VORTEILE FÜR BETRIEBSRÄTE}

Aus der Sicht einflussreicher Betriebsräte (insbesondere in Großbetriebsstrukturen) sind Öffnungsklauseln häufig erwünscht, eröffnen sie dem Gremium doch eine größere Teilhabe am Entscheidungsprozess und zusätzliche Möglichkeiten, Verhandlungsspielräume zu nutzen. Es stellt dabei sicher kein Problem dar, dass die Schlichtungsstelle als schärfstes Schwert des Betriebsrats nicht angerufen werden kann. Ihre Wirksamkeit erlangt diese Konfliktlösungseinrichtung vor allem durch die angedrohte Anrufung, was die notwendige Dynamik in den Verhandlungsprozess bringt (Brecht 2003, S. 141). Wenn schon die angedrohte Anrufung der Einigungsstelle wirksam ist, so muss die Androhung 
einer Rückverlagerung auf die tarifliche Ebene im Falle der Nichteinigung erst recht eine entsprechende Triebkraft entfalten. Zweifellos wird kein Betriebsrat von dieser Möglichkeit ohne Not Gebrauch machen, denn alle Beteiligten und auch die Mitarbeiter streben nach einer zeitnahen Lösung ohne die Kosten eines Arbeitskampfes.

Andererseits bestehen auch bei vielen Betriebsräten grundsätzliche Vorbehalte gegen eine Verlagerung auf die betriebliche Ebene. Die WSI-Betriebsrätebefragung von 2004/2005 ergab, dass immerhin $53 \%$ der Befragten eine Dezentralisierung und Verbetrieblichung der Tarifpolitik als grundsätzlich problematisch erachten (Schnabel 2006, S. 23f.). Dieser Umstand ist maßgeblich der Tatsache geschuldet, dass nicht wenige Betriebe eher eine Öffnung nach unten als nach oben anstreben. Auch die WSIBetriebsrätebefragung 2007 bestätigte, dass Betriebsräte sich mit einem zunehmend breiten Spektrum betrieblicher Entwicklungen konfrontiert sehen: Die Anforderungen sind in qualitativer wie in quantitativer Hinsicht beachtlich (Schäfer 2008, S. 293). Durch eine Verbetrieblichung von Tarifverhandlungen steigt die Belastung noch zusätzlich. Damit wächst zugleich das Konfliktpotenzial, wenn die Auseinandersetzungen auf die Betriebsebene verlegt werden (Schnabel 2006, S. 18f.).

Genau hier setzt jedoch die rückübertragbare Öffnungsklausel an. Denn am günstigsten ist es unter solchen Vorzeichen natürlich, wenn Öffnungsklauseln gar nicht erst notwendig werden. Wo sie dennoch praktikabel oder kaum vermeidbar sind, stellt die rückübertragbare Öffnungsklausel zumindest die verträglichste Variante dar. Im Vergleich zu "herkömmlichen“ Klauseln entlastet sie die Betriebspartner grundsätzlich. Denn wenn diese immer in der Gewissheit handeln, dass die Sozialpartner notfalls die „Kohlen aus dem Feuer“ holen, scheint die Ausgangslage von Anfang an entspannter. Die Betriebspartner können ihre bewährten Instrumente und Strukturen nutzen, um die für sie bestmögliche Lösung zu finden. Andererseits bleibt ihre Gesprächskultur unbeschädigt, falls sich keine Einigkeit einstellt. In diesem Fall würde die Normsetzungsaufgabe wieder zurück an die Tarifpartner gehen.

\subsection{VORTEILE FÜR UNTERNEHMEN}

Weniger einheitlich stellt sich die Interessenlage aus Sicht der Unternehmen dar, da das Verfahren mit Risiken im Falle der Nichteinigung verbunden ist. Zum einen könnte sich der Aushandlungsprozess entsprechend in die Länge ziehen. Im schlechtesten Fall wäre es möglich, dass es sowohl für die Flächenvereinbarung als auch für die betriebsindividuelle Lösung zu einem Arbeitskampf kommt. Dennoch ergeben sich gegenüber dem klassischen Flächentarifsystem oder selbst einem Aushandlungsprozess im reinen Ergänzungstarifvertrag auch für das Unternehmen mehr Vor- als Nachteile:

So ist die Verhandlungssituation für das Unternehmen im Vergleich zu einer Öffnungsklausel mit erzwingbarem Mitbestimmungsrecht eher kalkulierbar, da sich aufgrund vorangegangener Erfahrungen mit der zuständigen Gewerkschaft und der Belegschaft bessere Prognosen treffen lassen als über ein zu erwartendes Votum des Einigungsstellenvorsitzenden. Das Druckmittel einer angedrohten Rückverlagerung auf die tarifliche Ebene kann sich jedoch in bestimmten Konstellationen auch für den Arbeitgeber als nützlich erweisen, beispielsweise wenn eine von ihm angestrebte Regelung innerhalb des Korridors von Betriebsratsseite grundsätzlich abgelehnt wird. Hier kann ggf. durch einen - angedrohten oder tatsächlich herbeigeführten Wechsel der handelnden Akteure auf Arbeitnehmerseite neue Dynamik in den Verhandlungsprozess gebracht werden.

Schließlich haben die Unternehmen die Möglichkeit, maßgeschneiderte Lösungen für ihre spezielle Situation zu vereinbaren. Als Verhandlungspartner steht ihnen zunächst, wie dies häufig gewünscht wird, nicht die Gewerkschaft, sondern der eigene Betriebsrat gegenüber. Damit werden zugleich die Aussagen von Arbeitgeberseite einem Praxistest unterzogen, wonach Arbeitskämpfe überkommene Relikte darstellten und anachronistisch in einer Zeit seien, in der selbstbewusste Betriebsräte und ein sozial denkendes Management viel besser durch reinen Dialog einen Ausgleich finden könnten.

In der Summe lässt sich aus der Arbeitgebersicht sicher konstatieren, dass eine kombinierte Variante zwar weniger erstrebenswert erscheint als eine Öffnungsklausel klassischer Prägung. Gegenüber dem reinen Flächentarifsystem und selbst gegenüber dem Aushandlungsprozess im Rahmen eines Ergänzungstarifvertrages ergeben sich jedoch durchaus mehr Vorteile als Nachteile.

\subsection{VORTEILE DER TARIFVERTRAGSPARTEIEN}

Auch für die Tarifvertragsparteien wäre der Weg über die rückübertragbare Öffnungsklausel eine reizvolle Alternative. Dies gilt sowohl auf ordnungspolitischer wie auch auf verbands- und entgeltpolitischer Ebene.

Aus ordnungspolitischer Sicht würde vor allem das System des Flächentarifvertrages gestärkt. Zwar wird auch durch die rückübertragbare Öffnungsklausel die tarifliche Ordnungsfunktion partiell aufgehoben. Jedoch sind die Tarifparteien zweifellos stärker einbezogen, als dies bei unbeschränkten Öffnungsklauseln der Fall wäre. Wie bei allen Ansätzen zur kontrollierten Dezentralisierung steht dahinter der Gedanke, dass dort, wo eine Öffnung für betriebsindividuelle Lösungen erforderlich erscheint, diese dennoch innerhalb bestimmter Grenzen bzw. auch gesetzten Regeln gefunden werden soll.

Neben solchen weitgehend unbeschränkten Öffnungsklauseln ist in der Praxis nämlich auch das Interesse der Tarifparteien erkennbar, nach der Öffnung weiterhin mit in der Verantwortung zu bleiben. $\mathrm{Zu}$ diesem Zweck werden nicht elten Öffnungsklauseln formuliert, nach denen sich die Tarifpartner die letzte $\mathrm{Zu}$ stimmung vorbehalten. Eine derartige „konditionierte“ Öffnungsklausel haben beispielsweise die Partner der Chemieindustrie bereits 2000 abgeschlossen: Dort konnten Entgeltabweichungen durch die Betriebspartner vereinbart werden, jedoch behielten sich die Tarifparteien die Zustimmung vor (Bispinck/WSI-Tarifarchiv 2001, S. 14). Wegweisend war auch der sogenannte „Pforzheimer Abschluss“, bei dem die Metalltarifpartner - ihre Zustimmung vorausgesetzt - die Möglichkeit zur Unterschreitung von Tarifstandards erlauben, sofern dadurch Arbeitsplätze gesichert und geschaffen werden (Bispinck/WSI-Tarifarchiv 2007, S. 18).

Solch konditionierte Öffnungsklauseln erweisen sich als prinzipiell sinnvolle Regelungen, denn sie stabilisieren das System des Flächentarifvertrages. Dennoch ergeben rückübertragbare Öffnungsklauseln einen deutlichen Mehrwert: Zum einen sind Öffnungsklauseln mit Zustimmungsvorbehalt für die Tarifparteien mit einem nicht unerheblichen Prüfungsaufwand verbunden. Echte Öffnungen, welche absehbar in nur wenigen Fällen zu einer neuerlichen Befassung der Tarifpartner führen, stellen 
somit eine erhebliche Entlastung dar. Zum anderen wird mit einem Vorschlag der Betriebsparteien zur Abweichung vom Tarifvertrag ein erheblicher Zustimmungsdruck auf die Tarifpartner ausgeübt. Verweigern sie ihre Zustimmung, so bleibt es bei der Vereinbarung aus dem Flächentarifvertrag. Die Tarifparteien geraten dann leicht in Gefahr, als Blockierer für eine sachgerechte Lösung im Betrieb dargestellt zu werden. Meist wirkt sich dies nicht zuletzt auf die Mitgliederentwicklung negativ aus. Besteht hingegen aufgrund einer rückübertragbaren Öffnungsklausel die Möglichkeit, einen Arbeitskampf (wieder) zu eröffnen, erzeugt dies die notwendige Dynamik, um noch einmal Bewegung in einen festgefahrenen Verhandlungsprozess zu bringen.

Außerdem werden die Expertise sowie die offiziellen und inoffiziellen Kanäle und Instrumente der Betriebspartner wirksamer ausgenutzt, wenn diese selbst verhandeln statt „nur“ "konsultiert werden, wie das bei Haustarifverträgen geschieht. Auch der Forderung der Arbeitgeberseite, möglichst umfassend Regelungskompetenzen an die Betriebsparteien zu delegieren, würde so in einer regulierten Form umgesetzt - und letztlich besser, als dies bei den beschriebenen konditionierten Klauseln der Fall ist.

Die direkteste Beteiligung der Tarifvertragsparteien bestünde natürlich immer noch darin, betriebsbezogene Tarifverträge abzuschließen. So hat die IG Metall in der Bekleidungsindustrie eine Vereinbarung getroffen, nach der die Tarifparteien zum Zwecke der „nachhaltigen Verbesserung der Beschäftigungsentwicklung" selbst betriebsbezogene Ergänzungen vornehmen nach gemeinsamer Prüfung mit den Betriebsparteien (Bispinck/WSI-Tarifarchiv 2005, S. 9).

Solche Vereinbarungen treffen jedoch zum einen auf grundsätzliche Vorbehalte der Arbeitgeberverbände, welche stets auf direkte Entscheidungskompetenzen der Betriebsräte drängen. Zum anderen sind Haustarifverträge vor dem Hintergrund personeller Ressourcen vor allem dann attraktiv, solange sie nicht wirklich flächendeckend in jedem verbandsangehörigen Unternehmen einzeln ausgehandelt werden müssen. Der Arbeitsaufwand der Verbände ist vergleichsweise geringer, als wenn sofort und ohne Vorsortierung für jeden Betrieb ein Ergänzungstarifvertrag angestrebt würde. Gerade aus Gewerkschaftssicht wären massenhafte Haustarifverträge zumindest als problematisch zu werten.
Neben ordnungspolitische Motive treten verbandspolitischer Aspekte, denn ein Ziel der Tarifparteien ist natürlich, den eigenen Fortbestand zu sichern. Die vorgeschlagene Kombination aus Öffnungsklausel und Ergänzungstarifvertrag kommt den Tarifunterworfenen entgegen, was Verbandsflucht weniger attraktiv machen dürfte. Gleichzeitig behalten sich die Akteure weitgehende Rechte vor, anstatt durch eine endgültige Kompetenzverlagerung jeweils die eigene Position zu schmälern. Aber auch die Gewerkschaften können immer noch hervorragende Argumente für eine Verbandsmitgliedschaft geltend machen. Die Verhandlungsmacht sowohl der Betriebsräte als auch der Gewerkschaft resultiert wesentlich aus dem Organisationsgrad. Ein schwach organisierter Betrieb wird wohl selten mehr erreichen können als den Grundtarif der Fläche. Bei der herkömmlichen Öffnungsklausel hingegen entsteht bei den Arbeitnehmern hingegen eher der Eindruck, dass alles nur vom Geschick der Betriebsräte abhängt. Der Organisationsgrad ist dabei nur noch von untergeordneter Bedeutung.

Zuletzt können entgeltpolitische Ziele genannt werden. Immer wieder ertönt aus den Reihen der Betriebsräte Unmut darüber, dass Verteilungsspielräume nicht hinreichend ausgenutzt werden können. In anderen Betrieben mag wiederum auf wirtschaftliche Schieflagen mit einer zeitweisen Anpassung „nach unten“ reagiert werden. Das ist zwar auch bisher mit Haustarifverträgen oder mit Öffnungsklauseln möglich. Jedoch würde das Expertenwissen der betrieblichen Akteure erstmals institutionell mit den einander ebenbürtigen Druckmitteln der Tarifparteien verknüpft. Das schafft die besten Chancen, um einen angemessenen Anteil am Geschäftsergebnis für die Beschäftigten zu erlangen.

\section{Ergebnis}

Die Öffnung des Flächentarifvertrags für betriebliche Regelungen ist mit verschiedenen Risiken verbunden. Zum einen wird hierdurch die Ordnungswirkung des Tarifvertrags partiell aufgehoben, zum anderen handelt es sich bei den Betriebsparteien (anders als bei den Tarifvertragsparteien) nicht um strukturell gleich starke Verhandlungspartner.
Durch eine Weiterentwicklung bestehender tariflicher Regelungsmodelle lassen sich die bestehenden Probleme weiter minimieren. Vorgeschlagen wird eine „rückübertragbare Öffnungsklausel“: Den Betriebsparteien ist dabei im Rahmen eines eng vorgegebenen Korridors gestattet, von den tariflichen Regelvorgaben abzuweichen. Innerhalb einer bestimmten Zeitspanne können sie verhandeln und eine entsprechende Betriebsvereinbarung abschließen. Gelingt den Betriebsparteien keine Einigung, so fällt die Regelungskompetenz an die Tarifvertragsparteien zurück, die hinsichtlich des offen gelassenen Korridors keiner Friedenspflicht unterliegen.

Eine solche Tarifklausel ist rechtlich zulässig, da weder die negative Koalitionsfreiheit noch die tarifliche Ordnungsfunktion oder ein Selbstentäußerungsverbot für die Tarifvertragsparteien einer solchen Regelung entgegenstehen. Darüber hinaus liegt es aufgrund der Relativität der Friedenspflicht in der Gestaltungsfreiheit der Tarifvertragsparteien, das Arbeitskampfverbot für den Fall gescheiterter Verhandlungen auf Betriebsebene aufzuheben. Schließlich wird auch durch die Mitgliedschaft im Arbeitgeberverband die Möglichkeit eines firmenbezogenen Verbandstarifvertrags nicht ausgeschlossen.

Das vorgeschlagene Modell erweist sich aus Sicht der beteiligten Parteien als praktikabel: Starke Betriebsparteien erhalten den von ihnen gewünschten zusätzlichen Gestaltungsspielraum für betriebsindividuelle Lösungen, ohne insofern dem Druck ausgesetzt zu sein, unbedingt selbst zu einer Lösung gelangen zu müssen. Die mögliche Rückverlagerung auf die tarifliche Ebene bei Scheitern der betrieblichen Verhandlungen führt dabei zu einer erheblichen Dynamik, die im Regelfall zum Abschluss einer Betriebsvereinbarung führen dürfte. Für die Tarifvertragsparteien ist durch das Modell sichergestellt, dass sie ihre Einflussmöglichkeiten wahren und die tarifliche Ordnungswirkung nur soweit relativieren, wie dies von ihnen als notwendig betrachtet wird. Im Vergleich zur klassischen Öffnung des Tarifvertrags stellt die rückübertragbare Öffnungsklausel somit ein Modell dar, das eine wesentlich bessere Verzahnung betrieblicher und tariflicher Rechtsetzung ermöglicht. Es ist daher zu hoffen, dass die Tarifvertragsparteien zukünftig anstelle unbedingter tariflicher Öffnungen das Regelungsinstrument der rückübertragbaren Öffnungsklausel wählen. 
Bauer, J.-H./Krieger, S. (2004): „Firmentarifsozialplan“ als zulässiges Ziel eines Arbeitskampfes?, in: Neue Zeitschrift für Arbeitsrecht Nr, S. 10191021

Baumann, T. (1992): Die Delegation tariflicher Rechtsetzungsbefugnisse, Berlin

Bispinck, R./WSI-Tarifarchiv (2001): Tarifpolitischer Jahresbericht 2000. Moderate Lohnabschlüsse plus „Beschäftigungsbrücke“, Düsseldorf Bispinck, R./WSI-Tarifarchiv (2005): Wie flexibel sind Tarifverträge? Eine Untersuchung von Tarifverträgen in über 20 Wirtschaftszweigen und Tarifbereichen, Elemente qualitativer Tarifpolitik 60, Düsseldorf Bispinck, R./WSI-Tarifarchiv (2007): Tarifpolitischer Jahresbericht 2006. Aufwärtstrend bei Tarifabschlüssen - Konflikte um Beschäftigungssicherung und Tarifstandards, Düsseldorf

Brecht, H. (2003): Die Umsetzung von Tarifverträgen auf Betriebsebene. Das Verhältnis betrieblicher und tariflicher Rechtsetzung auf der Grundlage rechtstatsächlicher Erkenntnisse, Berlin

Brecht-Heitzmann, H. (2007): Verhinderung von Betriebsstillegung durch Sozialtarifvertrag?, in: Neue Juristische Wochenschrift Nr, S. 3617-3620 Buchner, H. (1970): Möglichkeiten und Grenzen betriebsnaher Tarifpolitik, Teil II, in: Der Betrieb Nr, S. 2074-2077

Gragert, N. (1997): Möglichkeiten und Grenzen der Flexibilisierung von Tarifverträgen zugunsten betrieblicher Regelungen, Hamburg

Kempen O./Zachert U. (2006): Tarifvertragsgesetz, Frankfurt am Main
Krichel, U. (1986): Ist der Tarifvertrag mit einem verbandsangehörigen Arbeitgeber erstreikbar?, in: Neue Zeitschrift für Arbeitsrecht Nr, S. 731736

Lobinger, T. (2006): Streiks um unternehmensbezogene Tarifverträge bei Verbandstarifgebundenheit des Arbeitgebers - Zur Wiedergeburt der "betriebsnahen Tarifpolitik“ im modernen Standortkampf, in: Recht der Arbeit Nr, S. 12-22

Matthes, H. (1998): Tarifautonomie für ein neues Jahrhundert, in: Ascheid, R./Friedrich H./Schlachter M. (Hrsg): Festschrift für Günter Schaub, München, S. 477-485

Ohl, K./Meine H./Lang K. (1997): Arbeit, Entgelt, Leistung. Handbuch Tarifarbeit im Betrieb, Köln

Richardi, R./Wlotzke, O. (2000): Münchner Handbuch zum Arbeitsrecht, München

Schäfer, C. (2008): Die WSI-Betriebsrätebefragung 2007 - Methoden und ausgewählte Ergebnisse, in: WSI-Mitteilungen 6, S. 291-298 Schnabel, C. (2006): Verbetrieblichung der Lohnfindung und der Festlegung von Arbeitsbedingungen, Hans Böckler Stiftung, Arbeitspapier 118, Düsseldorf

Schwarze, R. (1990): Der Betriebsrat im Dienst der Tarifvertragsparteien, Berlin

Wendeling-Schröder, U. (1998): Betriebliche Ergänzungstarifverträge, in: Neue Zeitschrift für Arbeitsrecht Nr, S. 624-630 Wiedemann, H. (2007): Tarifvertragsgesetz, München 


\section{ZUSAMMENFASSUNGEN}

BRECHT-HEITZMANN, HOLGER/GRÖLS, MARCEL

Betriebsnahe Tarifpolitik durch rückübertragbare Öffnungsklauseln

in: WSI-Mitteilungen 9/2008, Seiten

Die Öffnung des Flächentarifvertrags für betriebliche Regelungen ist mit Risiken verbunden. Zum einen wird hierdurch die Ordnungswirkung des Tarifvertrags partiell aufgehoben, zum anderen handelt es sich bei den Betriebsparteien nicht um strukturell gleich starke Verhandlungspartner. Vorgeschlagen wird daher eine Weiterentwicklung tariflicher Regelungen durch eine "rückübertragbare Öffnungsklausel“" Den Betriebsparteien ist dabei im Rahmen eines Korridors gestattet, von den tariflichen Regelvorgaben abzuweichen. Innerhalb einer bestimmten Zeit können sie eine entsprechende Betriebsvereinbarung abschließen. Gelingt den Betriebsparteien keine Einigung, so fällt die Regelungskompetenz an die Tarifvertragsparteien zurück, die hinsichtlich des offen gelassenen Korridors keiner Friedenspflicht unterliegen. Nach einer umfassenden Prüfung wird die rechtliche Zulässigkeit einer solchen Regelung bejaht. Ferner wird dargestellt, dass sich eine solche Klausel aus der Sicht der beteiligten Parteien als praktikabel und attraktiv erweist, da sie eine wesentlich bessere Verzahnung betrieblicher und tariflicher Rechtsetzung ermöglicht. 